\title{
Value of surgical resection compared to transarterial chemoembolization in the treatment of hepatocellular carcinoma with portal vein tumor thrombus: A meta-analysis of hazard ratios from five observational studies
}

\author{
Keera Kang, Sung Kyu Song, Chul-Woon Chung, and Yongkeun Park
}

\author{
Department of Surgery, Catholic Kwandong University International St. Mary's Hospital, Incheon, Korea
}

\begin{abstract}
Backgrounds/Aims: Although systemic therapy is recommended in advanced hepatocellular carcinoma (HCC), treatment options for advanced HCC with portal vein tumor thrombosis (PVTT) are debatable. Recent studies have recommended other treatments, such as surgical resection (SR) and transarterial chemoembolization (TACE). Therefore, we performed a meta-analysis of hazard ratio (HR) for overall survival (OS) between the two modalities using previous reports in order to compare the two treatment options. Methods: A systematic review was performed on previously reported data that compared the survival benefits of SR and TACE in patients with advanced HCC with PVTT. Thereafter, the meta-analysis was performed to determine the cumulative HR between the two different treatment groups. We used the HR and $95 \% \mathrm{Cl}$ directly from the original data, when available; however, if these data were unavailable, reconstruction was performed with the secondary data from the original Kaplan-Meier survival curve. Results: A total of seven studies were eligible; however, 2 were excluded from the meta-analysis. The remaining 5 studies that included 1422 patients (SR group=559, TACE group=863) were studied for the meta-analysis. The median OS was longer in the SR group (8.2-64 months in SR vs. 6.6-32 months in TACE), proving that SR offered survival benefits. Moreover, the HR for the OS in the TACE group was $1.64(95 \% \mathrm{Cl}, 1.43-1.88)$ compared to SR group, depicting that TACE was a less favorable option compared to SR. Conclusions: There is evidence that SR may be a better viable option for advanced HCC with PVTT. (Ann Hepatobiliary Pancreat Surg 2020;24:243-251)
\end{abstract}

Key Words: Hepatectomy; Hepatic resection; Portal vein invasion; Kaplan-Meier survival data

\section{INTRODUCTION}

Hepatocellular carcinoma (HCC) is one of the most frequently diagnosed malignancies worldwide. Its incidence has been increasing annually, making it one of the leading causes of cancer-related deaths. Various factors, such as chronic hepatitis B/C infections, advanced age, obesity, alcohol abuse, diabetics, and family history are associated with a higher risk of HCC. ${ }^{1}$ The Barcelona Clinic Liver Cancer (BCLC) system is one of the most renowned staging systems for $\mathrm{HCC}$ that has provided a guideline for HCC treatment. In the early stages of HCC, interventions with surgical resection (SR), transplantation, and local ablations are recommended and are known to benefit the patient. In addition, in the stage of $\mathrm{HCC}$, transarterial chemoembolization (TACE) is also helpful. In the advanced stages of HCC, systemic therapy with sorafenib is considered the treatment of choice. However, its effect appears limited because it improves the patients' overall survival (OS) only by few months. ${ }^{2-4}$

In advanced HCC, patients either have macrovascular invasion or extrahepatic spread, with poor prognosis. In particular, HCC with portal vein tumor thrombosis (PVTT) is believed to be a negative prognostic factor because it increases the likelihood of tumor cell spread into the bloodstreams, resulting in higher chances of recurrence. ${ }^{5}$ Previous studies have stated that HCC patients with portal vein invasion have a median survival period of 2.7-4 months

Received: May 4, 2020; Revised: July 13, 2020; Accepted: July 13, 2020

Corresponding author: Yongkeun Park

Department of Surgery, Catholic Kwandong University International St. Mary's Hospital, Catholic Kwandong University College of Medicine, 25 Simgokro 100-gil, Seo-gu, Incheon 22711, Korea

Tel: +82-32-290-3912, Fax: +82-32-290-3879, E-mail: ykpark@ish.ac.kr

Copyright (C) 2020 by The Korean Association of Hepato-Biliary-Pancreatic Surgery

This is an Open Access article distributed under the terms of the Creative Commons Attribution Non-Commercial License (http://creativecommons.org/ licenses/by-nc/4.0) which permits unrestricted non-commercial use, distribution, and reproduction in any medium, provided the original work is properly cited. Annals of Hepato-Biliary-Pancreatic Surgery - pISSN: 2508-5778 - elSSN: 2508-5859 
without any treatment. ${ }^{6,7}$ However, as per recent studies, depending on the patient's baseline performance status, hepatic function, tumor characteristics, the type of therapy could increase the survival duration from 5 months to 5 years. ${ }^{8,9}$ Therefore, it is crucial to choose the optimal treatment. As briefly mentioned above, sorafenib was usually recommended. However, with continuous research, other treatments, such as SR and TACE, are available options for treating advanced HCC. ${ }^{10,11}$

$\mathrm{SR}$ is the main treatment option in early HCC and is indicated when there is a solitary tumor in patients without cirrhosis. However, several studies have stated that SR is a possible treatment modality in HCC patients with PVTT, particularly for patients with type I or II PVTT. ${ }^{12,13}$ TACE is the procedure of choice for intermediate stage HCC. Some studies have shown survival benefits in asymptomatic patients with multifocal disease without vascular invasion or extrahepatic spread. It is believed not to be beneficial in portal vein invasion and it is especially contraindicated in PVTT type III and IV because this can lead to necrosis of the hepatic tissue. However, in few studies, TACE appears to be a treatment option in HCC patients with PVTT. ${ }^{14,15}$

Therefore, it is crucial to evaluate and recognize whether SR or TACE is beneficial in advanced HCC with PVTT and which modality offers more advantages. This will in turn provide other treatment options in addition to sorafenib for patients with advanced HCC with PVTT. Thus, recent studies have compared these treatment modalities in advanced HCC with PVTT. To our knowledge, no randomized-controlled trial has been reported, while some recent meta-analysis studies have focused on this research area. In previous meta-analysis studies, the intervention effect was measured with calculated odds ratios (OR) with the standard error from the original studies. ${ }^{16,17}$ Using this method, time-to-event data was analyzed as dichotomous data. However, the hazard ratio (HR), usually calculated from a Cox proportional hazards model, is the most appropriate way for analyzing survival end points in studies of intervention effect. ${ }^{18}$ Thus, we aimed to perform a meta-analysis of HR for overall survival (OS) between two treatment modalities groups. In order to obtain an estimate of the HR, when it is not reported in original studies, the Kaplan-Meier (KM) data were reconstructed from the published survival curves of each study.

\section{MATERIALS AND METHODS}

A systematic review of studies reporting on survival, comparing SR to TACE in patients with HCC with PVTT was performed. We exclusively abstracted and then combined in a meta-analysis the HRs from the observational studies. All study-specific estimates were combined using inverse variance-weighted averages of logarithmic HRs in both fixed- and random-effects models. This analysis was conducted following the Preferred Reporting Items for Systematic Reviews and Meta-Analyses (PRISMA).

\section{Literature search}

Electronic searches were performed using MEDLINE, Cochrane Library, and KoreaMed, from their date of inception to December 2019. To obtain the maximum sensitivity of the search strategy and identify all studies, we combined "carcinoma, hepatocellular" as Medical Subject Headings (MeSH) terms or keywords with each of "portal vein" AND "hepatectomy" or "liver resection" or "surgical resection" AND "Transarterial chemoembolization" or "TACE". We also searched reviews about similar issues via manual screening of the reference lists for further identification of potentially relevant studies. Titles and abstracts of retrieved articles were examined to exclude irrelevant reports. For further screening, all the selected articles were systematically assessed using the inclusion and exclusion criteria independently by two investigators (KK and SKS). Disagreements were subjected to discussions for reaching a consensus.

\section{Eligibility criteria}

Studies with assessable reports on the survival comparisons in HCC patients with PVTT who underwent SR and TACE were included and were judged suitable for the meta-analysis only if the HR between two treatment modalities or the figures of Kaplan-Meier survival curves were available. When institutions published several similar studies with accumulating data of the involved patients, the most complete reports were used for our final analyses. Studies with sample size $<20$ for each treatment modality group were excluded. Only publications in English language were considered. Abstracts, case reports, conference presentations, editorials, and expert opinions were excluded because of potential publication bias and 
result duplication. Primary end points of the study were the assessment of cumulative HR between the two treatment modalities groups.

\section{Survival data extraction and reconstruction of Kaplan-Meier data}

Data regarding the author's first name, publication year, study methods, patient characteristic, interventions, and outcomes were extracted and assessed by two investigators (KK and SKS). We extracted and recorded the HR and $95 \%$ CI directly from the original data whenever available. If not, we reconstructed the KM survival data for each treatment group from the published survival curves. The time and survival probability coordinates were extracted from the figures of survival curves using the DigitizeIt software (www.digitizeit.de). We extracted the numbers of patients at risk and the total numbers of events in each treatment group from the text, when available. The KM survival data were reconstructed using a unique algorithm introduced by Guyot et al. ${ }^{19}$ that was adopted to inversely solve the $\mathrm{KM}$ equations.

\section{Quality assessment}

The quality assessment of the included studies was as- sessed using the modified Newcastle-Ottawa Scale (NOS) that included selection, comparability, and exposure.

\section{Comparison of the survival outcomes according to $\mathrm{HR}$}

The reconstructed patient survival data of each treatment group were then aggregated to form combined KM data for estimating the HR and associated variance using a Cox proportional hazards regression model. Endpoints in this meta-analysis were evaluated with HRs and 95\% CIs using both fixed- and random-effects model.

\section{Statistical analyses}

For survival analysis and HR calculation, "survival" package in $\mathrm{R}$ was used. Meta-analyses were performed using "meta" package in $\mathrm{R}^{20}$ The significance of the pooled HR was evaluated using the $Z$ test $(p<0.05$ was considered as statistically significant). The heterogeneity across studies was assessed using the I2 statistic. The I2 statistic was derived from the $\mathrm{Q}$ statistic that provides a measure of the proportion of the overall variation attributable to heterogeneity between the studies. Heterogeneity was considered significant if the $\mathrm{Q}$ statistic showed $p<0.05$ and when the I2 statistic exceeded $50 \%{ }^{21}$

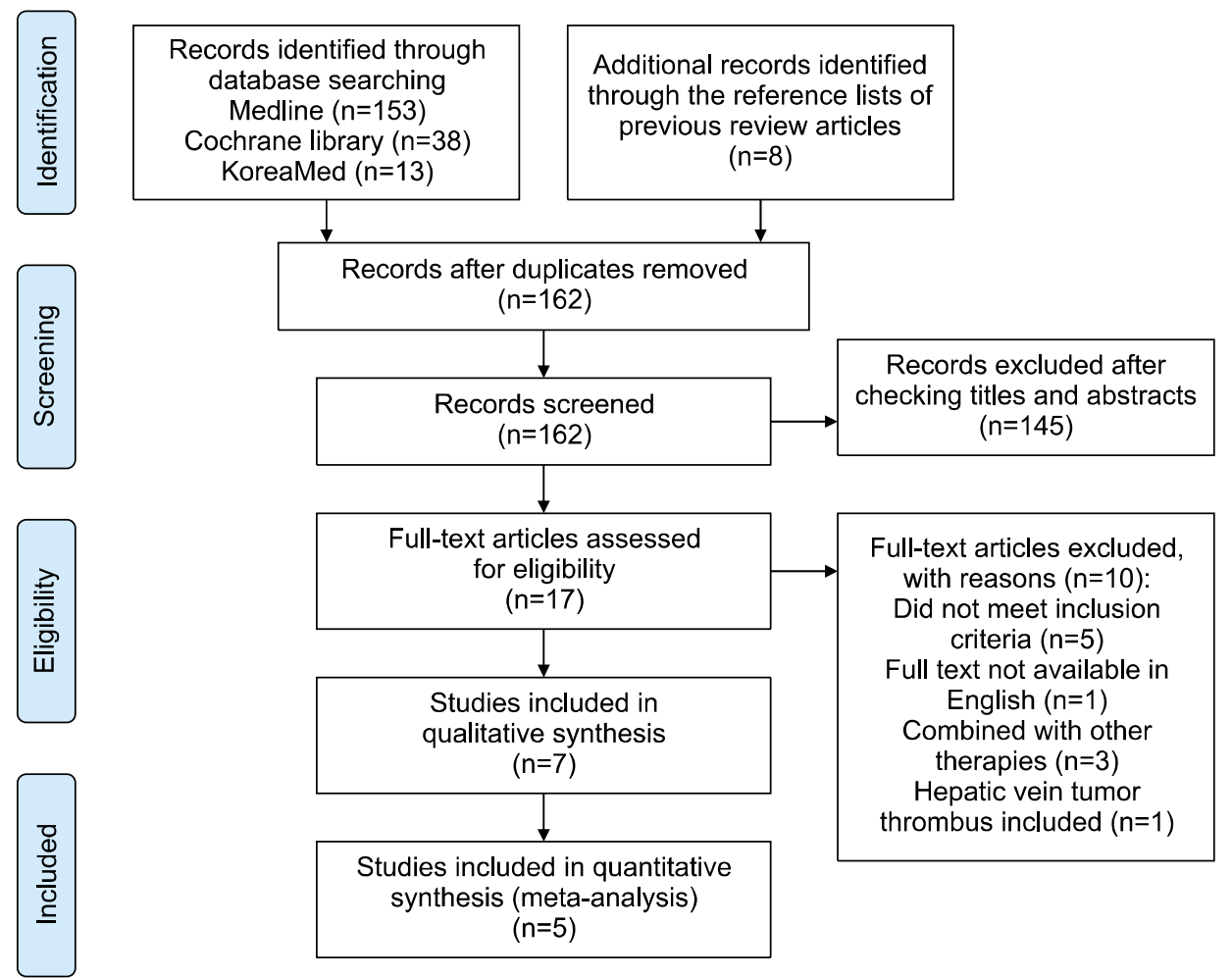

Fig. 1. Flow chart for study selection. 


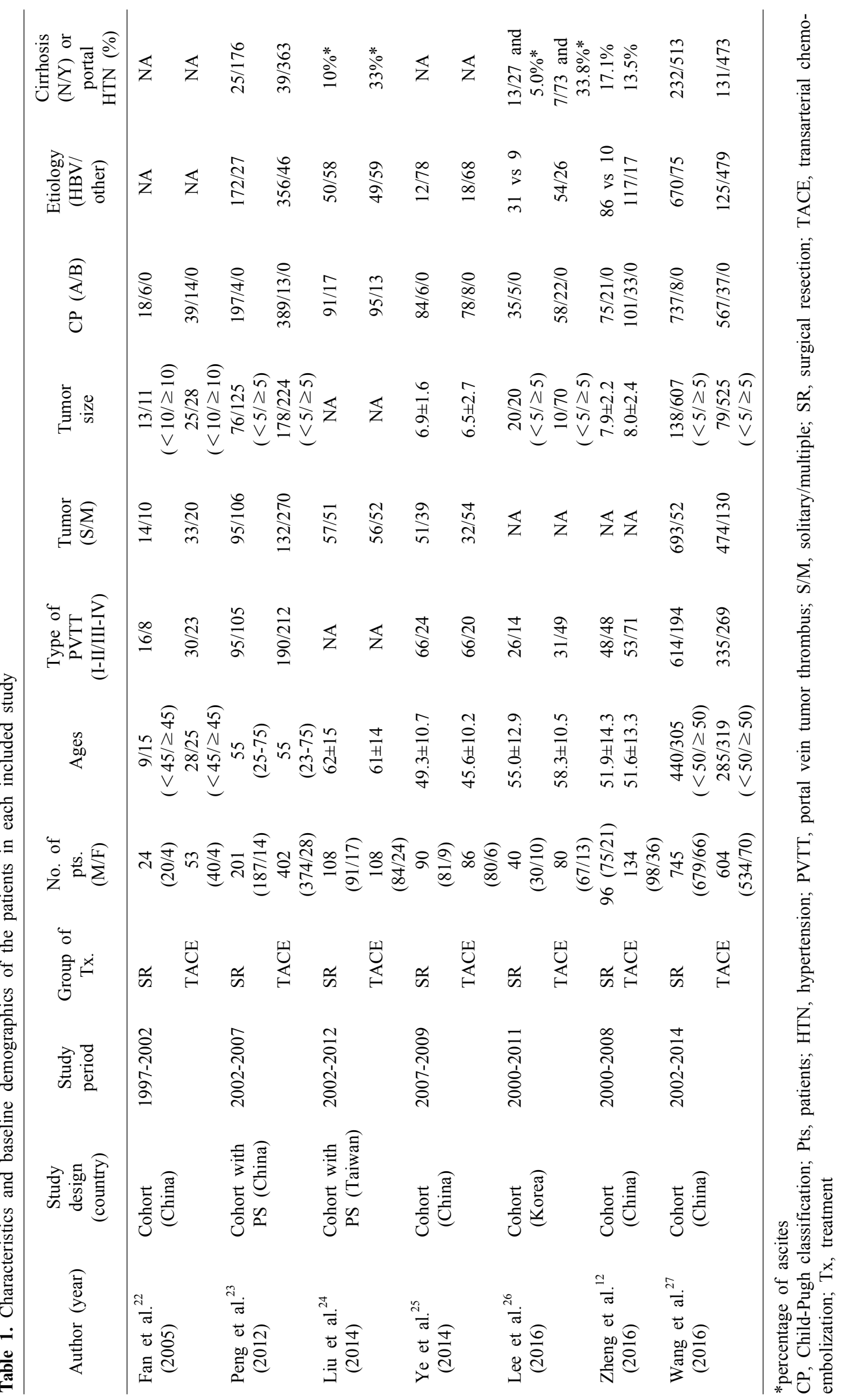




\section{RESULTS}

\section{Characteristics of the included studies}

In total, 162 non-duplicate records were retrieved. After screening of titles and abstracts, 145 records were excluded and 17 records were assessed for eligibility. The full text of these articles was read, seven retrospective cohort studies ${ }^{12,22-27}$ meeting eligibility criteria were included in the systematic review (Fig. 1). The characteristics and baseline demographic data of the patients in each research study are listed in Table 1 . The recruitment period ranged from 1997 to 2012. No prospective studies were found during the search. All studies were from Asia, including one from Korea, one from Taiwan, and five from China. Two studies ${ }^{22,27}$ was excluded from the meta-analysis because the reconstruction of survival data from original KM graph was not possible because no KM survival graph was reported or there were inadequate data of the numbers of patients at risk and the total numbers of events (death). The remaining 5 studies including 1422 patients were finally included in this meta-analysis. The groups were classified as follows: 559 patients in the SR group and 863 patients in the TACE group. Patients enrolled in the systematic review were within Child-Pugh class A or $\mathrm{B}$ in all the studies. No study reported statistically significant differences in the baseline demographic or clinical and tumor characteristics of the two groups. The NOS scores of the included studies ranged from 5-7 and were considered of high quality: 5 studies ${ }^{12,23-25,27}$ were scored 6-7 and $2^{22,26}$ were scored 5 .

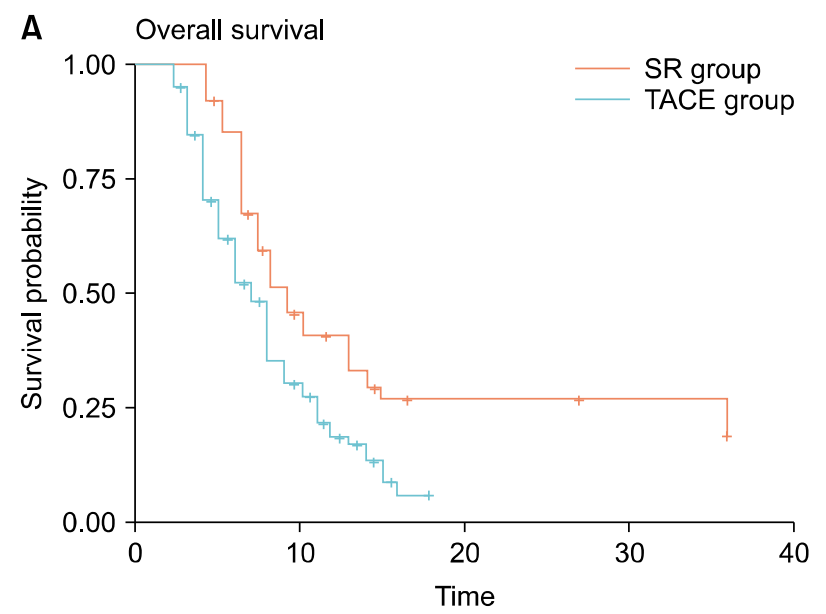

\section{Comparison of the OS in the SR group and TACE group}

In the enrolled studies, the median OS ranged from 8.2-64 months in the SR group and 6.6-32 months in the TACE group. In the SR group, the 1-year OS rate ranged from $22.7 \%-86.5 \%$, the 3 -year OS rate ranged from $0 \%-69 \%$, and the 5 -year OS rate ranged from $0 \%-69 \%$. In the TACE group, the 1-year OS rate was $11.8 \%-77.6 \%$, the 3 -year OS rate was $0 \%-50 \%$, and the 5 -year OS rate was $0.5 \%-35 \%$. Based on the original survival data reported in the enrolled studies, the 1-, 3-, and 5-year OS rates were worse for patients receiving TACE than those for patients undergoing SR.

\section{Hazard ratios}

To compare the survival outcomes between SR and TACE group, the HR was defined as the ratio of risk of death in the TACE group over the risk of death in the SR group. The HRs of OS could be extracted directly from the original data in three studies, ${ }^{23,24,26}$ however, two studies $^{12,25}$ did not show HR or $95 \%$ CI directly in the original paper. To obtain an estimate of the HR from the latter two studies, the Kaplan-Meier data were reconstructed from the published survival curves of each study. Each KM curve of SR and TACE group was extracted separately. Using the algorithm provided by Guyot et al., ${ }^{19}$ the reconstructed KM survival data were obtained that enabled us to reconstruct the KM curves and was used to acquire a reconstructed HR (Fig. 2). With full KM data, the reconstructed HR of one study ${ }^{25}$ was $1.922(95 \%$

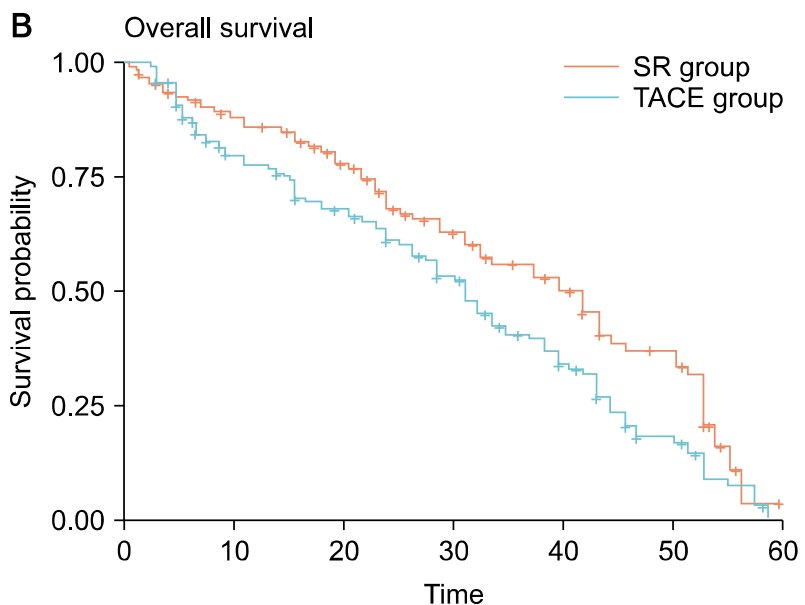

Fig. 2. Reconstructed Kaplan-Meier (KM) survival curves of 2 studies ((A) Ye et al., ${ }^{25}$ (B) Zheng et al. ${ }^{12}$ ) to acquire hazard ratios secondarily from original $\mathrm{KM}$ data using the algorithm of Guyot et al. ${ }^{19}$ 

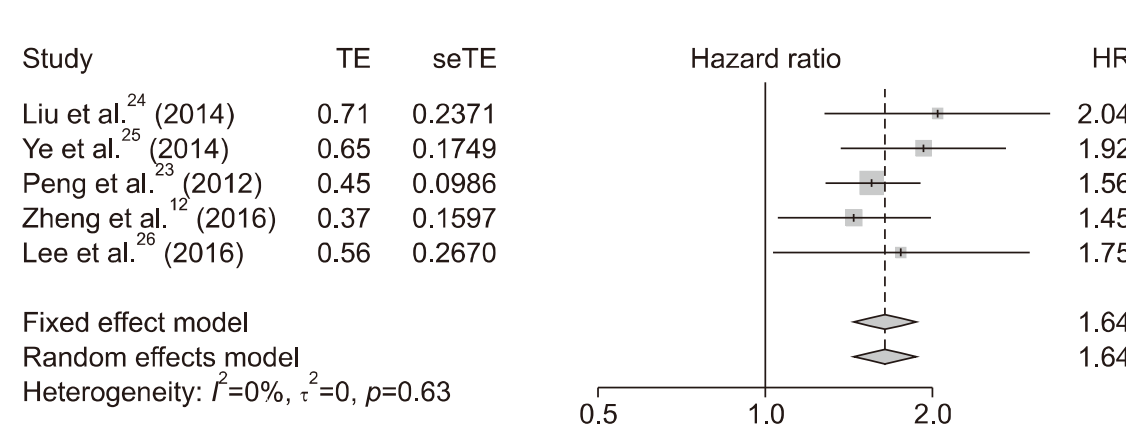

$\begin{array}{rrrr}\text { HR } & 95 \% \mathrm{Cl} & \begin{array}{r}\text { Weight } \\ \text { (fixed) }\end{array} & \begin{array}{r}\text { Weight } \\ \text { (random) }\end{array} \\ 2.04 & {[1.28 ; 3.25]} & 8.6 \% & 8.6 \% \\ 1.92 & {[1.36 ; 2.71]} & 15.8 \% & 15.8 \% \\ 1.56 & {[1.29 ; 1.90]} & 49.8 \% & 49.8 \% \\ 1.45 & {[1.06 ; 1.98]} & 19.0 \% & 19.0 \% \\ 1.75 & {[1.04 ; 2.95]} & 6.8 \% & 6.8 \% \\ & & & \\ 1.64 & 1.64[1.43 ; 1.88] & 100.0 \% & -- \\ 1.64 & 1.64[1.43 ; 1.88] & -- & 100.0 \%\end{array}$

Fig. 3. Forest plot depicting hazard ratio of overall survival in transarterial chemoembolization compared to surgical resection using fixed-effect and random-effect models.

CI, 1.364-2.708) and that of the other study $^{12}$ was 1.447 (95\% CI, 1.058-1.979).

\section{Endpoints}

The OS were compared between the SR group and TACE groups and were evaluated in five of the included studies. Using both the fixed-effect and random-effect models, the pooled HR for the OS in the TACE group was 1.64 (95\% CI, 1.43-1.88, Fig. 3) compared to that in the SR group. Heterogeneity was negligible among these studies.

\section{Publication bias analysis}

Publication bias analysis was conducted for comparing the primary endpoint between the SR group and the TACE group. There was no apparent publication bias on visual inspection of the funnel plot of the standard error with $\log$ HR for overall survival (Fig. 4) or with Egger's test $(p=0.266)$.

\section{DISCUSSION}

In clinical oncology, survival analysis is particularly vital because most cancer researches investigate time-to -event endpoints, such as OS and recurrence-free survival. It is also a fundamental aspect of decision-making regarding treatment options and is used to interpret the efficacy of interventions. Survival data are unique in that usually not all patients experience the event by the end of the observation period. For some patients, their actual survival periods are not given. Censoring or incompletely observed survival times are inherent in these data. Survival times of patients may also be skewed; therefore, the use of stat-

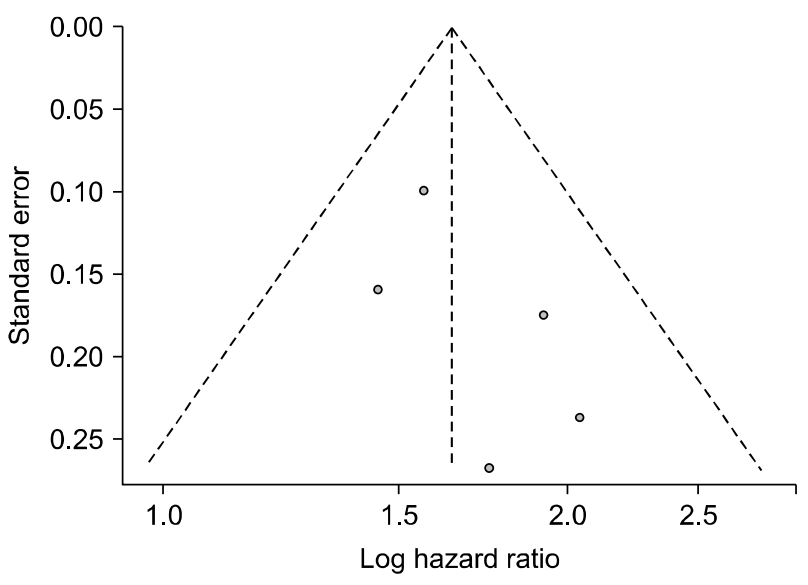

Fig. 4. Funnel plot depicting the publication bias in the included studies.

istical methods that assume a normal data distribution is limited. Therefore, special statistical techniques are needed to compare the survival between the two groups. The risk ratio (relative risk) and OR are relative measures, and are appropriate for measuring dichotomous outcomes. However, they measure only the number of events and neglect the time of occurrence. Thus, they are not appropriate for analyzing time-to-event outcomes. However, $\mathrm{HR}$ is usually provided in a Cox proportional hazards model that considers the number and timing of events, and the time until last follow-up for patients without the event. Thus, it is an appropriate method for time-to-event analysis or survival analysis over the entire study duration. $^{28,29}$

The aim of our meta-analysis was to compare two treatments option (SR and TACE) for HCC patients with PVTT in terms of overall survival. In order to obtain a more robust and reliable estimate of patient survival, we performed a meta-analysis of HRs. Most meta-analyses 
combine the data retrieved from different comparative studies with a single endpoint to produce a single pooled result. In this kind of meta-analysis, outcomes are reported as binomial proportions, and the most popular measure of effect is the OR. As mentioned in the previous paragraph, the HR is an appropriate measure in comparing survival analysis, and therefore should be considered a natural quantity when undertaking a meta-analysis of survival studies. Nevertheless, there is a limitation to using HRs in meta-analysis when data regarding HRs are not given. Fortunately, an alternative approach has been proposed when HRs are not available in original articles. ${ }^{19}$ Image extraction software (www.digitizeit.de) is used to extract the coordinates of Kaplan-Meier (KM) curves from the original articles. The numbers at risk at follow-up times were calculated using number at risk tables. An algorithm is applied to reconstruct the survival data that are then re-analyzed to report the HR and its respective $95 \%$ CI. We utilized this method to obtain HRs from the KM survival curves of two studies (Fig. 2). The calculated HRs were used in the meta-analysis of HRs with original reported HRs from three other studies. This alternative approach appears valuable to reduce the publication bias in meta-analysis when HRs are not reported in the original studies.

In many cases of advanced $\mathrm{HCC}$, there is a simultaneous presence of PVTT. Therefore, in these cases, the surgical procedures may differ, depending on where the tumor thrombosis exists. Tumor thrombectomy can be performed when PVTT does not involve the bifurcation of the portal vein and the tumor thrombus is also contained in the same lobe of the liver as the HCC lesion itself. However, tumor thrombectomy involves the risk of residual tumor. A different surgical technique is recommended when PVTT extends to the portal vein or further, such as en-bloc resection with reconstruction of the portal vein. This technique involves full resection of the vessel containing the tumor thrombus and reconstructing the remaining portal vein in an end-to-end anastomosis manner. This method is complex; therefore, it has higher morbidity and mortality rates but is believed to achieve better oncological outcomes. However, in several studies, tumor thrombectomy had similar survival outcomes with lower operative mortality and morbidity. ${ }^{30-32}$ Therefore, it is difficult to determine which procedure is better than the oth- er because as per our literature search, there is lack of studies comparing the two different kinds of surgery.

As briefly discussed in the beginning of the article, there are several other treatment options for advanced HCC that have not been included in our study but deserve to be mentioned. Sorafenib, a molecular target agent, has been regarded as the main treatment for advanced HCC. The Sorafenib HCC Assessment Randomized Protocol (SHARP) is a randomized-controlled trial that was conducted in the United States and Europe. In this study, the median overall survival was 10.7 months in the group treated with sorafenib, while that in the control group (placebo group) was 6.9 months. Moreover, the time to progression significantly differed by 5.5 months in the sorafenib group versus 2.9 months in the control group. However, another study on an Asia-Pacific population reported a median OS of 6.5 months for the sorafenib group. ${ }^{3,33-36}$ Another option proposed in recent studies is radioembolization where microspheres containing a specific type of radioactive material are injected into the hepatic artery that becomes entrapped and selectively emits high-dose radiation to the HCC lesion. One study in Italy showed a median OS of 15 months. ${ }^{37}$ Another trial with fewer advanced HCC patients in Korea showed a 3-year survival rate of 50\%-75\%. ${ }^{38}$ However, these treatment options have many diverse adverse effects that may interfere with the patients' consistent compliance to these therapies compared to surgical therapy.

One limitation of our study was that it was based on the secondary data obtained from original papers. Therefore, information on important perioperative variables, such as antiviral drug use and underlying liver disease progression, was not available. These are believed to contribute to tumor recurrence and de novo malignancy. Also, other important considerations in the selection of SR, such as liver function which was only compared by using the Child-Pugh classification in our study, the presence of portal hypertension or low platelet count which reflects the presence of portal hypertension and remnant liver volume which were not available in some of the selected articles could not be accurately assessed in our study. In addition, the present systematic review and meta-analysis included only retrospective studies that are subject to inherent selection bias. The trial also included the geological limitation of enrolled studies that were all con- 
ducted in Eastern Asia and hepatitis B virus-endemic areas. Therefore, further prospective studies on a global level, which include the mentioned limitations above, are necessary.

Our findings showed that SR is a superior treatment option compared to TACE for treating HCC patients with PVTT. Thus, the evidence from these accumulated recent studies that justify the use of SR as a treatment option in advanced HCC patients with PVTT should be used as a stepping stone to assist modifications in the future guidelines for recommended therapies in advanced HCC with PVTT, especially in Eastern Asia. Furthermore, additional intricately designed prospective studies are in need to fully reinforce these findings.

\section{ORCID}

Keera Kang: https://orcid.org/0000-0002-7276-5980

Sung Kyu Song: https://orcid.org/0000-0003-0170-0802

Chul-Woon Chung:

https://orcid.org/0000-0003-0873-567X

Yongkeun Park: https://orcid.org/0000-0001-9481-6542

\section{AUTHOR CONTRIBUTIONS}

All authors have contributed significantly: KK, YP. Designed the study: KK, SKS, YP. Analysed and interpreted data: KK, SKS, CWC, YP. Wrote and/or reviewed the manuscript: all authors discussed the results and comment on the manuscript.

\section{REFERENCES}

1. Forner A, Reig M, Bruix J. Hepatocellular carcinoma. Lancet 2018;391:1301-1314.

2. Tunissiolli NM, Castanhole-Nunes MMU, Biselli-Chicote PM, Pavarino EC, da Silva RF, da Silva RC, et al. Hepatocellular carcinoma: a comprehensive review of biomarkers, clinical aspects, and therapy. Asian Pac J Cancer Prev 2017;18:863-872.

3. Llovet JM, Ricci S, Mazzaferro V, Hilgard P, Gane E, Blanc JF, et al. Sorafenib in advanced hepatocellular carcinoma. N Engl J Med 2008;359:378-390.

4. Cheng AL, Kang YK, Chen Z, Tsao CJ, Qin S, Kim JS, et al. Efficacy and safety of sorafenib in patients in the Asia-Pacific region with advanced hepatocellular carcinoma: a phase III randomised, double-blind, placebo-controlled trial. Lancet Oncol 2009;10:25-34.

5. Cerrito L, Annicchiarico BE, Iezzi R, Gasbarrini A, Pompili M, Ponziani FR. Treatment of hepatocellular carcinoma in patients with portal vein tumor thrombosis: beyond the known frontiers. World J Gastroenterol 2019;25:4360-4382.

6. Minagawa M, Makuuchi M. Treatment of hepatocellular carcinoma accompanied by portal vein tumor thrombus. World J Gastroenterol 2006;12:7561-7567.

7. Llovet JM, Bustamante J, Castells A, Vilana R, Ayuso Mdel C, Sala $\mathrm{M}$, et al. Natural history of untreated nonsurgical hepatocellular carcinoma: rationale for the design and evaluation of therapeutic trials. Hepatology 1999;29:62-67.

8. Manzano-Robleda Mdel C, Barranco-Fragoso B, Uribe M, Méndez-Sánchez N. Portal vein thrombosis: what is new? Ann Hepatol 2015;14:20-27.

9. Suh SJ, Yim HJ, Lee DW, Hyun JJ, Jung YK, Kim JH, et al. Factors affecting prognosis of hepatocellular carcinoma with portal vein tumor thrombosis: implications for future therapeutic strategies. J Liver Cancer 2017;17:60-71.

10. Jarnagin W, Chapman WC, Curley S, D'Angelica M, Rosen C, Dixon E, et al. Surgical treatment of hepatocellular carcinoma: expert consensus statement. HPB (Oxford) 2010;12:302-310.

11. Kudo M, Izumi $\mathrm{N}$, Kokudo $\mathrm{N}$, Matsui O, Sakamoto $\mathrm{M}$, Nakashima O, et al. Management of hepatocellular carcinoma in Japan: Consensus-Based Clinical Practice Guidelines proposed by the Japan Society of Hepatology (JSH) 2010 updated version. Dig Dis 2011;29:339-364.

12. Zheng N, Wei X, Zhang D, Chai W, Che M, Wang J, et al. Hepatic resection or transarterial chemoembolization for hepatocellular carcinoma with portal vein tumor thrombus. Medicine (Baltimore) 2016;95:e3959.

13. Shi J, Lai EC, Li N, Guo WX, Xue J, Lau WY, et al. Surgical treatment of hepatocellular carcinoma with portal vein tumor thrombus. Ann Surg Oncol 2010;17:2073-2080.

14. Liu L, Zhang C, Zhao Y, Qi X, Chen H, Bai W, et al. Transarterial chemoembolization for the treatment of advanced hepatocellular carcinoma with portal vein tumor thrombosis: prognostic factors in a single-center study of 188 patients. Biomed Res Int 2014;2014:194278.

15. Silva JP, Berger NG, Tsai S, Christians KK, Clarke CN, Mogal $\mathrm{H}$, et al. Transarterial chemoembolization in hepatocellular carcinoma with portal vein tumor thrombosis: a systematic review and meta-analysis. HPB (Oxford) 2017;19:659-666.

16. Zhang XP, Wang K, Li N, Zhong CQ, Wei XB, Cheng YQ, et al. Survival benefit of hepatic resection versus transarterial chemoembolization for hepatocellular carcinoma with portal vein tumor thrombus: a systematic review and meta-analysis. BMC Cancer 2017;17:902.

17. Zhang ZY, Dong KS, Zhang EL, Zhang LW, Chen XP, Dong $\mathrm{HH}$. Resection might be a meaningful choice for hepatocellular carcinoma with portal vein thrombosis: a systematic review and meta-analysis. Medicine (Baltimore) 2019;98:e18362.

18. Batson S, Greenall G, Hudson P. Review of the reporting of survival analyses within randomised controlled trials and the implications for meta-analysis. PLoS One 2016;11:e0154870.

19. Guyot P, Ades AE, Ouwens MJ, Welton NJ. Enhanced secondary analysis of survival data: reconstructing the data from published Kaplan-Meier survival curves. BMC Med Res Methodol 2012;12:9.

20. R Development Core Team. R: a language and environment for statistical computing. Vienna: R Foundation for Statistical Computing; 2017. Available from: https:/www.R-project.org/.

21. Higgins JP, Thompson SG, Deeks JJ, Altman DG. Measuring inconsistency in meta-analyses. BMJ 2003;327:557-560.

22. Fan J, Zhou J, Wu ZQ, Qiu SJ, Wang XY, Shi YH, et al. Efficacy of different treatment strategies for hepatocellular carcinoma with portal vein tumor thrombosis. World J Gastroenterol 
2005; 11:1215-1219.

23. Peng ZW, Guo RP, Zhang YJ, Lin XJ, Chen MS, Lau WY. Hepatic resection versus transcatheter arterial chemoembolization for the treatment of hepatocellular carcinoma with portal vein tumor thrombus. Cancer 2012;118:4725-4736.

24. Liu PH, Lee YH, Hsia CY, Hsu CY, Huang YH, Chiou YY, et al. Surgical resection versus transarterial chemoembolization for hepatocellular carcinoma with portal vein tumor thrombosis: a propensity score analysis. Ann Surg Oncol 2014;21:1825-1833.

25. Ye JZ, Zhang YQ, Ye HH, Bai T, Ma L, Xiang BD, et al. Appropriate treatment strategies improve survival of hepatocellular carcinoma patients with portal vein tumor thrombus. World J Gastroenterol 2014;20:17141-17147.

26. Lee JM, Jang BK, Lee YJ, Choi WY, Choi SM, Chung WJ, et al. Survival outcomes of hepatic resection compared with transarterial chemoembolization or sorafenib for hepatocellular carcinoma with portal vein tumor thrombosis. Clin Mol Hepatol 2016;22:160-167.

27. Wang K, Guo WX, Chen MS, Mao YL, Sun BC, Shi J, et al. Multimodality treatment for hepatocellular carcinoma with portal vein tumor thrombus: a large-scale, multicenter, propensity mathching score analysis. Medicine (Baltimore) 2016;95:e3015.

28. Lee ET, Go OT. Survival analysis in public health research. Annu Rev Public Health 1997;18:105-134.

29. Schober P, Vetter TR. Survival analysis and interpretation of time-to-event data: the tortoise and the hare. Anesth Analg 2018; 127:792-798.

30. Tanaka A, Morimoto T, Yamaoka Y. Implications of surgical treatment for advanced hepatocellular carcinoma with tumor thrombi in the portal vein. Hepatogastroenterology 1996;43:637-643. 31. Chok KS, Cheung TT, Chan SC, Poon RT, Fan ST, Lo CM. Surgical outcomes in hepatocellular carcinoma patients with portal vein tumor thrombosis. World J Surg 2014;38:490-496.

32. Wu CC, Hsieh SR, Chen JT, Ho WL, Lin MC, Yeh DC, et al. An appraisal of liver and portal vein resection for hepatocellular carcinoma with tumor thrombi extending to portal bifurcation. Arch Surg 2000;135:1273-1279.

33. Song MJ, Bae SH. Newer treatments for advanced hepatocellular carcinoma. Korean J Intern Med 2014;29:149-155.

34. Cheng AL, Guan Z, Chen Z, Tsao CJ, Qin S, Kim JS, et al. Efficacy and safety of sorafenib in patients with advanced hepatocellular carcinoma according to baseline status: subset analyses of the phase III Sorafenib Asia-Pacific trial. Eur J Cancer 2012;48:1452-1465.

35. Kudo M. Signaling pathway and molecular-targeted therapy for hepatocellular carcinoma. Dig Dis 2011;29:289-302.

36. Wilhelm SM, Adnane L, Newell P, Villanueva A, Llovet JM, Lynch M. Preclinical overview of sorafenib, a multikinase inhibitor that targets both Raf and VEGF and PDGF receptor tyrosine kinase signaling. Mol Cancer Ther 2008;7:3129-3140.

37. Mazzaferro V, Sposito C, Bhoori S, Romito R, Chiesa C, Morosi $\mathrm{C}$, et al. Yttrium- 90 radioembolization for intermediate-advanced hepatocellular carcinoma: a phase 2 study. Hepatology 2013;57: 1826-1837.

38. Kim DY, Park BJ, Kim YH, Han KH, Cho SB, Cho KR, et al. Radioembolization with yttrium-90 resin microspheres in hepatocellular carcinoma: a multicenter prospective study. Am J Clin Oncol 2015;38:495-501. 